\title{
Splicing factors differentially expressed in psoriasis alter mRNA maturation of disease-associated EDA+ fibronectin
}

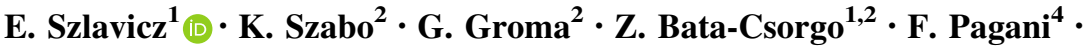 \\ L. Kemeny ${ }^{1,2} \cdot$ M. Szell ${ }^{2,3}$
}

Received: 17 February 2017/ Accepted: 1 June 2017/Published online: 6 June 2017

(C) Springer Science+Business Media, LLC 2017

\begin{abstract}
The EDA+ fibronectin splicing variant is overexpressed in psoriatic non-lesional epidermis and sensitizes keratinocytes to mitogenic signals. However, regulation of its abundance is only partially understood. In our recent cDNA microarray experiment, we identified three SR-rich splicing factors-splicing factor, arginine/serine-rich 18 (SFRS18), peptidyl-prolyl cis-trans isomerase G (PPIG), and luc-7 like protein 3 (LUC7L3)—which might be implicated in the preactivated states of keratinocytes in psoriatic non-involved skin and could also contribute to the regulation of fibronectin mRNA maturation. In this study, we investigated the role of LUC7L3, PPIG, and SFRS18 in psoriasis and in the mRNA maturation process of fibronectin. Regarding tissue staining experiments, we were able to demonstrate a characteristic distribution of the splicing factors in healthy, psoriatic non-involved and involved epidermis. Moreover, the expression profiles of these SR-rich proteins were found to be very similar in synchronized keratinocytes. Contribution of splicing
\end{abstract}

Electronic supplementary material The online version of this article (doi:10.1007/s11010-017-3090-1) contains supplementary material, which is available to authorized users.

E. Szlavicz

szlavicz.eszter@gmail.com

1 Department of Dermatology and Allergology, Faculty of Medicine, University of Szeged, 6 Korányi fasor, Szeged 6720, Hungary

2 MTA-SZTE Dermatological Research Group, Szeged, Hungary

3 Department of Medical Genetics, Faculty of Medicine, University of Szeged, Szeged, Hungary

4 International Centre for Genetic Engineering and Biotechnology, Trieste, Italy facwwtors to the EDA+ fibronectin formation was also confirmed: their siRNA silencing leads to altered fibronectin mRNA and protein expression patterns, suggesting the participation in the EDA domain inclusion. Our results indicate that LUC7L3, PPIG, and SFRS18 are not only implicated in EDA+ fibronectin formation, but also that they could possess multiple roles in psoriasis-associated molecular abnormalities.

Keywords Psoriasis - mRNA splicing · EDA+ fibronectin $\cdot$ SR-rich splicing factors

\section{Introduction}

During the past few years, huge advances have been made in the field of psoriasis research; however, the exact molecular background of this chronic inflammatory disorder has not been fully revealed and many questions remain to be answered. Development of psoriatic lesions is associated with keratinocyte hyperproliferation and abnormal T-lymphocyte function, although it is still controversial whether epithelial or immunological elements are determinant in the primary phase of the disease. The extent to which genetic and environmental factors lead to the disorder is also a subject of discussion [1-3].

Recently, a novel molecular factor has been identified that might be responsible for the elevated responsiveness of keratinocytes to the stimuli of professional immune cells. This putative factor is a splice variant of fibronectin that contains the oncofetal EDA domain (EDA+ fibronectin) [4-6]. Although fibronectin is an excellent model for studying splicing (having more than 20 mRNA variants), processes regulating mRNA maturation and the mechanisms affecting EDA+ fibronectin formation are not fully 
understood [7, 8]. The EDA domain is a Type III repetitive module of fibronectin, typically present during embryonic development. Although the tendency is exclusion of the EDA domain in the adult organism, EDA+ fibronectin is elevated under some circumstances, including wound healing, inflammation, and malignant processes [8-13]. In psoriasis, elevated EDA+ fibronectin expression was described for psoriatic non-involved epidermis, and we showed that proliferating keratinocytes are able to produce this isoform $[4,5]$.

Several molecular factors in addition to EDA+ fibronectin have been implicated in psoriasis pathogenesis. Application of large-scale gene expression studies extended our knowledge regarding molecular abnormalities in involved and non-involved epidermis [14-19]. Recent RNASequencing-based experiments have indicated that a large number of mRNAs are consistently differentially expressed in healthy and involved skin samples across independent studies, at both the transcript and gene levels [20, 21]. Specifically, a systematic comparison of the above-mentioned datasets indicated over 10,000 differentially expressed transcripts, suggesting the potential role of mRNA maturation processes in psoriasis development [22].

In a recently performed cDNA microarray experiment, we aimed to compare the responsiveness of healthy and psoriatic non-involved epidermis to treatment with a mixture of T-lymphokines (GM-CSF, IFN- $\gamma$, IL-3) previously shown to induce psoriatic plaque formation [23, 24]. Functional characterization of the identified genes exhibiting differential regulation upon T-lymphokine treatment showed that they play a role in several cellular procedures including the influence of cell morphology, development, and cell death. Moreover, a set of these genes participate in the metabolism of small lipids [23]. Among these genes were three that are implicated in mRNA maturation: splicing factor, arginine/serine-rich 18 (SFRS18, also known as PNN-interacting serine/arginine-rich protein, PNISR), peptidyl-prolyl cis-trans isomerase $G$ (PPIG), and luc-7 like protein 3 (LUC7L3). Products of these genes belong to the family of SR-rich proteins and function as trans-regulators of splicing events (UniProtKBO95232 (LC7L3_HUMAN); UniProtKB-Q13427 (PPIG_HUMAN); UniProtKB-Q8TF01 (PNISR_HUMAN)). This finding is notable, as relatively few articles mention the relationship between psoriasis and disturbances of mRNA maturation.

In our study, characteristics of splicing-factor expression were examined in synchronized, immortalized cell cultures and skin samples. Since the regulation of fibronectin splicing is only partially elucidated, we aimed to determine whether these newly identified splicing factors participate in the production of the EDA+ fibronectin isoform. For this purpose, an in vitro functional assay was constructed to detect changes in fibronectin splicing patterns during decreased expression of the LUC7L3, PPIG, and SFRS18 splicing factors.

\section{Materials and methods}

\section{Skin biopsies}

To carry out our cDNA microarray experiment, 4 healthy volunteers and 4 patients (age 18-60 years) with moderateto-severe chronic plaque-type psoriasis (Psoriasis Area and Severity Index score determined by dermatologists) were enrolled in the study after a medication-free period of $\geq 4$ weeks without systemic therapy and/or $\geq 2$ weeks without local therapy. Prior to surgical intervention, participant-informed consent was obtained. Tissue collection complied with the guidelines of the Declaration of Helsinki and was approved by the Regional and Institutional Research Ethics Committee (2799, 3517).

\section{Organotypic skin cultures}

Organotypic skin cultures were established from each halfcut from shave biopsies [23]. Briefly, epidermis samples placed on cellulose acetate/cellulose nitrate filters $(2.2 \mu \mathrm{m}$ porosity, Millipore) were transferred to a stainless steel grid platform in a 6-well plate. As culture medium, Dulbecco's modified Eagle's medium (DMEM) supplemented with $12 \mathrm{mM}$ glutamine, 100 units $/ \mathrm{ml}$ penicillin, and $100 \mu \mathrm{g} / \mathrm{ml}$ streptomycin (all from Life Technologies, Carlsbad, CA) was applied. For all donor skin specimens, one-half of the organotypic cultures was treated with the lymphokine mixture, containing $1 \mathrm{ng} / \mathrm{ml}$ IFN $\gamma, 1 \mathrm{ng} / \mathrm{ml}$

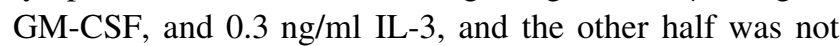
treated. Using standard culturing conditions (at $37{ }^{\circ} \mathrm{C}$ in a $5 \% \mathrm{CO}_{2}$ atmosphere), organotypic skin cultures were maintained at the air/liquid interface for $72 \mathrm{~h}$. Subsequently, the epidermis was separated from the dermis by overnight incubation in Dispase solution (grade II, Roche Applied Science) at $4{ }^{\circ} \mathrm{C}$, and then placed in TRI Reagent (Molecular Research Center Inc., Cincinnati, OH).

\section{Real-time RT-PCR}

One microgram of total RNA purified from organotypic skin culture samples, HPV-KER and $\mathrm{HaCaT}$ cells were reverse transcribed using the iScript TM cDNA Synthesis kit (\#1708891, Bio-Rad, Hercules, CA). RT-PCR was performed with the cDNA to quantify transcript abundance using custom primer sets and the Universal Probe Library (Roche, Basel, Switzerland) with an iQ Supermix (\#1708862, Bio-Rad, Hercules, CA). Relative gene expression data were calculated by normalizing the 
expression data for the $18 \mathrm{~S}$ ribosomal RNA and using the $\Delta \Delta \mathrm{Ct}$ method.

\section{Immunofluorescent staining}

Biopsies were frozen, embedded, stored at $-80{ }^{\circ} \mathrm{C}$, and then cut into $6-\mu \mathrm{m}$ sections. The following primary antibodies were used: LUC7L3 (1:300, Abcam, Cambridge, UK), PPIG (1:300, Abcam), and SFRS18 (1:250, Novus Biologicals, Littleton, USA). Anti-mouse IgG-Alexa Fluor 647 and anti-rabbit Alexa Fluor 546 were used as secondary antibodies, both at 1:500 dilution (Life Technologies, Carlsbad, CA). For negative staining controls, sections were incubated without the primary antibody (in case of PPIG) or with normal rabbit IgG as isotype control antibody (Santa Cruz Biotechnology, Dallas, TX, USA). Nuclei were stained with 2-(4-amidinophenyl)-1H-indole6-carboxamidine (DAPI).

Fluorescent microscopic images were analyzed using ImageJ software, by calculating corrected-total-cell-fluorescence values based on integrated density of stained cell areas.

\section{Culturing of HPV-KER and HaCaT cells}

The HPV-KER cell line was immortalized by the HPV E6 oncogene as described by our research group [25]. HPVKER cells were grown in $75 \mathrm{~cm}^{2}$ cell culture flasks and maintained in keratinocyte serum-free medium $\left(\mathrm{Gibco}^{\circledR}\right.$ Keratinocyte SFM Kit; Life Technologies, Copenhagen, Denmark) supplemented with $1 \%$ antibiotic/antimycotic solution (PAA, Pasching, Austria) and 1\% L-glutamine (PAA) at $37{ }^{\circ} \mathrm{C}$ in a humidified atmosphere containing $5 \%$ $\mathrm{CO}_{2}$. The medium was changed every two days.

During synchronization of the HPV-KER cells and after confluence was obtained, cells were maintained in glutamine-free and growth factor-free keratinocyte SFM for one week. Cells were harvested with brief trypsinization $(0.25 \%$ trypsin solution Sigma Aldrich, St. Louis, MO, USA)

Immortalized HaCaT keratinocytes were kindly provided by Dr. Fusenig, N.E. (Heidelberg, Germany). Cells were synchronized as described previously [26].

\section{Western blot analysis}

For western blot analysis, equal amounts of proteins were separated on a $10 \%$ SDS-PAGE gel and transferred to Pure Nitrocellulose Membrane (Bio-Rad Laboratories). Primary antibodies were the following: LUC7L3 (1:300, Abcam, Cambridge, UK), PPIG (1:300, Abcam), and SFRS18 (1:300, Novus Biologicals, Littleton, US). Anti-mouse and anti-rabbit IgG alkaline phosphate conjugate (Sigma Aldrich, St. Louis, MO, USA) was used as a secondary antibody, and signals were visualized with Sigma Fast TM BCIP/NBT (Sigma Aldrich, St. Louis, MO, USA). An $\alpha$ actin-specific antibody was used as a loading control (1:2000, Sigma Aldrich, St. Louis, MO, USA).

\section{Gene-specific silencing}

Gene-specific silencing was performed with an siRNA transfection method. HPV-KER cells were transiently transfected at approximately $70 \%$ confluency. siRNA silencing (transfection reagent and medium) was ordered from Santa Cruz Biotechnology (Dallas, TX, USA). In silencing experiments, scrambled and gene-specific LUC7L3, PPIG, SFRS18 siRNA duplexes were applied. The most effective silencing was achieved in serum-free culture medium without additive and supplements. The effectiveness of silencing was confirmed by real-time RT-PCR.

\section{Polymerase chain reaction}

Samples were collected from the cultures of silenced HPVKER cells. Total RNA was isolated from cell cultures by TRIzol ${ }^{\circledR}$ Reagent (Invitrogen Corp., Carlsbad, CA, USA), following the instructions provided in the manual. cDNA was synthesized from $1 \mu \mathrm{g}$ total RNA with the iScript cDNA Synthesis Kit (Bio-Rad Laboratories, Hercules, CA, USA). Primers specific for human fibronectin (5'-AAGCCAATTTCCATTAATTACCGAAC- $3^{\prime}$ and 5'-TCTCATACT TGATGATGTAGCCGGTAA- $3^{\prime}$ ) were used to amplify a $1221 \mathrm{bp}$ product from the EDA+ template and an 847 bp product from the EDA - fibronectin template. PCR conditions are described in Szell et al., and detection and semiquantitative analysis of the differentially spliced fibronectin form are also demonstrated in this paper [4]. Ten microliters of the PCR products were run on $1 \%$ agarose gel, photographed and evaluated using Bio-Rad Gel Doc XR densitometer.

\section{Flow cytometry}

Cells were collected after a brief trypsinization $(0.25 \%$ trypsin solution Sigma Aldrich, St. Louis, MO, USA), fixed in Fixation/Permeabilization Concentrate and Diluent (eBioscience, San Diego, USA), and resuspended in PBS. Primary antibodies (anti-EDA+-fibronectin, anti-fibronectin, 1:500 and 1:1000, respectively, Sigma Aldrich, St. Louis, MO, USA) were applied for $45 \mathrm{~min}$. Cells were then washed in PBS and incubated with anti-mouse IgGAlexa Fluor 647 (Life Technologies, Carlsbad, CA) as secondary antibody, at 1:500 dilution. Samples were analyzed on a FACSCalibur flow cytometer equipped with 488 and 633 nm lasers (Becton-Dickinson, Franklin Lakes, NJ, USA). 


\section{Statistical analysis and bioinformatics}

Statistical analysis was performed in GraphPad Prism 5.0 Software (GraphPad Prism, San Diego, CA), using onesample $t$ - and unpaired $t$-tests with a $p<0.05$ significance threshold.

Predictive interaction analysis was carried out using the publicly available STRING database (http://string-db.org/).

\section{Results}

\section{Differential expression of splicing-factor genes confirmed for psoriasis}

LUC7L3, PPIG, and SFRS18 splicing regulators have been identified in our above-mentioned cDNA microarray experiment. An overview of our approach is presented in Fig. 1.a. Briefly, gene expression before and after lymphokine treatment was compared in healthy epidermis, and we applied the same approach to non-involved psoriatic skin. Genes exhibiting at least a two-fold change in relative expression have been chosen for further comparison. We then selected the genes showing significant differences in mean expression between the control and psoriatic noninvolved samples [23]. Real-time reverse-transcription polymerase chain reaction (RT-PCR) was carried out to verify whether the identified genes are differentially regulated in psoriasis. The T-lymphokine induced alterations were confirmed in the case of both LUC7L3 and PPIG splicing factors (Fig. 1b) Although we were not able to validate the cDNA microarray results for SFRS18 in the RT-PCR experiment, we decided to include this gene in further experiments as it participates in similar biological processes as $L U C 7 L 3$ and $P P I G$.

Expression of the splicing factors was also compared in untreated healthy and psoriatic non-involved epidermis samples of the microarray experiment: in this setup, the expression of LUC7L3 and SFRS18 was slightly higher in psoriatic non-involved epidermis than in healthy samples (Fig. 1c).

\section{Differentially expressed splicing factors in psoriasis}

As the splicing factors showing altered mRNA expression in psoriasis are poorly characterized, we examined the pattern of protein expression in healthy, psoriatic non-involved and involved skin samples. LUC7L3 and PPIG exhibited nuclear localization, whereas SFRS18 showed perinuclear staining (Fig. 2a). LUC7L3 is present in significantly higher amounts in psoriatic non-involved
Fig. 1 Experimental design and comparisons used in our previous cDNA microarray study is summarized in (a). Gene expression changes of LUC7L3, PPIG, and SFRS18 splicing factors induced by T-lymphokine treatment were compared in healthy $(\mathrm{H}, n=4)$ and psoriatic non-involved (PS, $n=4)$ epidermis samples (b), where the average expression level of untreated samples was considered as baseline expression (relative unit of 1 indicated by dashed line). Comparison of $L U C 7 L 3, P P I G$, and SFRS18 basal mRNA expression levels in untreated healthy $(\mathrm{H}, n=4)$ and psoriatic non-involved (PS, $n=4)$ epidermis. $p<0.05$ was considered significant (c) epidermis, whereas SFRS18 shows only minimal, nonsignificant elevation in non-involved epidermis. The highest levels of expression of LUC7L3 and SFRS18 were found in psoriatic involved epidermis samples. The expression pattern of PPIG was different from that of the other splicing factors: PPIG amounts were significantly lower in psoriatic non-involved epidermis than in healthy and psoriatic samples; however-similarly to LUC7L3 and SFRS18 - the highest expression was also detected in psoriatic involved epidermis (Fig. 2b).

\section{LUC7L3, PPIG, and SFRS18 expression patterns in synchronized cells}

To compare the expression levels in proliferating and differentiating keratinocytes, both mRNA and protein expression patterns of the selected splicing factors were analyzed in synchronized, immortalized cells. HPV-KER cells were synchronized by contact inhibition and in the absence of growth factors [22]. Cells were harvested at various time points after release from cell quiescence, and RNA and protein levels were measured by real-time RTPCR and western blot analysis, respectively.

Comparing the expression of the splicing factors during the proliferation and differentiation phases, a very similar mRNA expression pattern was observed for all three splicing factors (Fig. 3a). mRNA expression decreased at $12 \mathrm{~h}$. The first peak of mRNA abundance was seen at $24 \mathrm{~h}$, with steady elevation starting from $48 \mathrm{~h}$, a phenomenon that is likely induced by cellular stress processes. This synchronization experiment was repeated using synchronized, immortalized $\mathrm{HaCaT}$ cells: the splicing regulators exhibited an identical pattern of gene expression in $\mathrm{HaCaT}$ cells that were observed in the HPV-keratinocytes (Suppl. Fig. 1)

The pattern of expression in HPV-KER cells of both RNA and protein was similar. However, protein expression was more even across sampled time points than for mRNA. The highest expression of LUC7L3, PPIG, and SFRS18 protein was detected at $48 \mathrm{~h}$ after release from cell quiescence (Fig. 3b). 
(A)
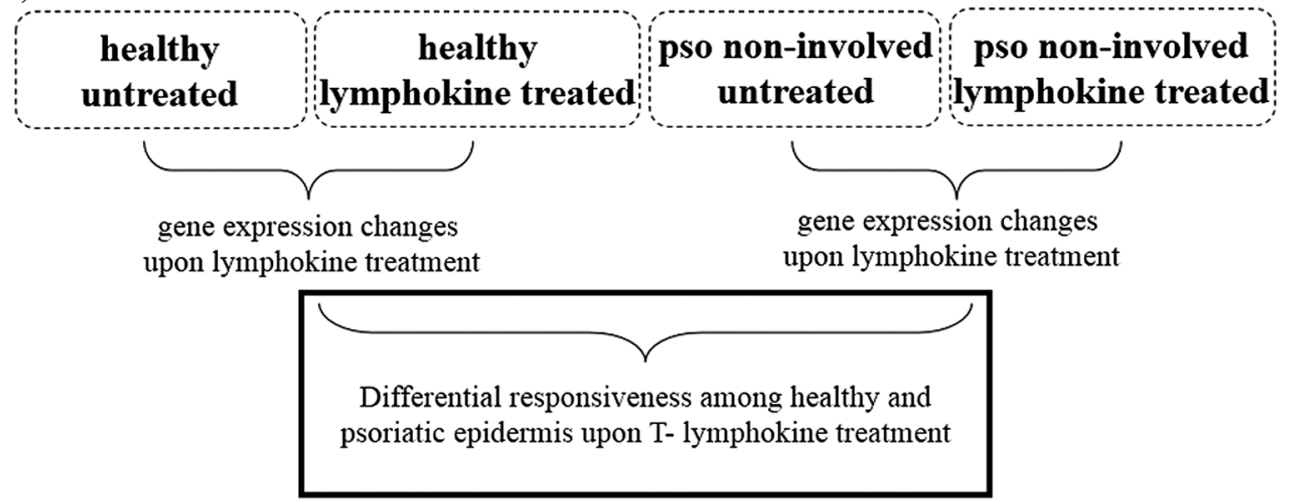

Fold-change $>2, p<0.05,95 \% \mathrm{CI}$

(B)
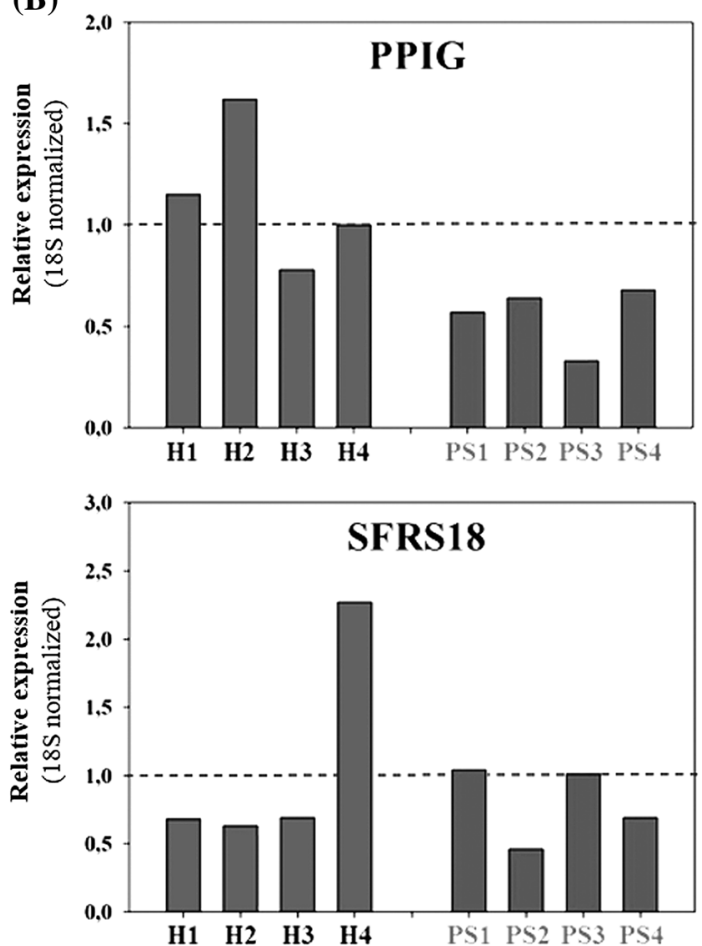

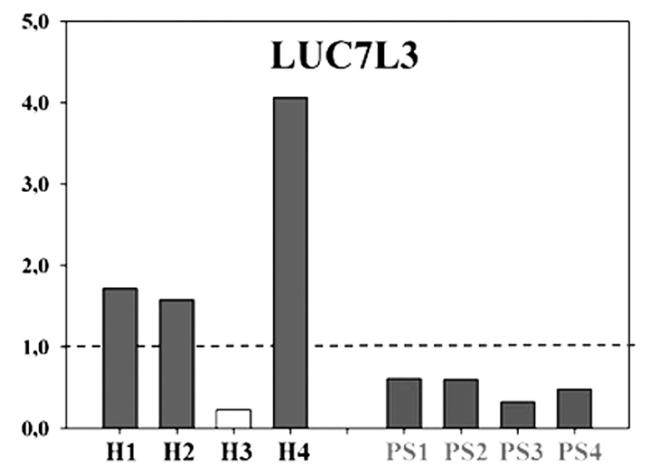

(C)

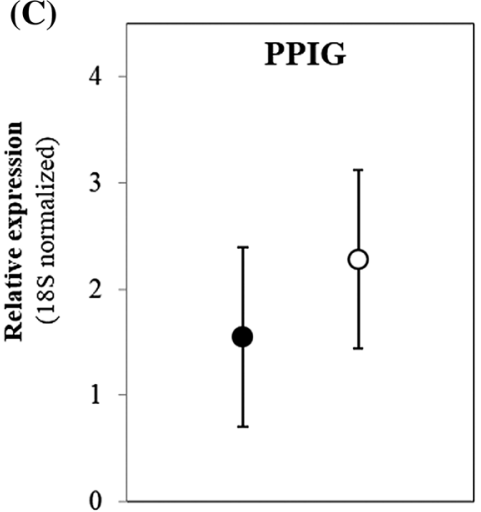

H PS

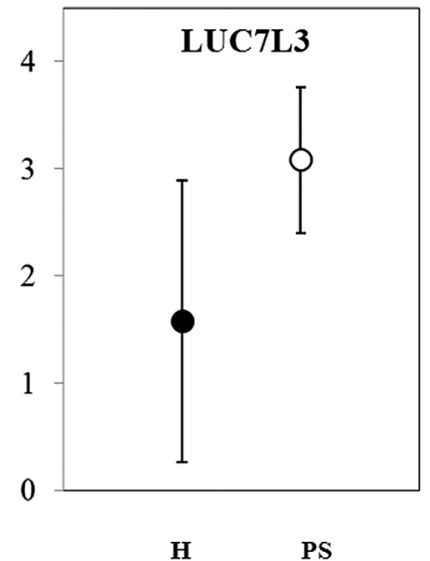

H

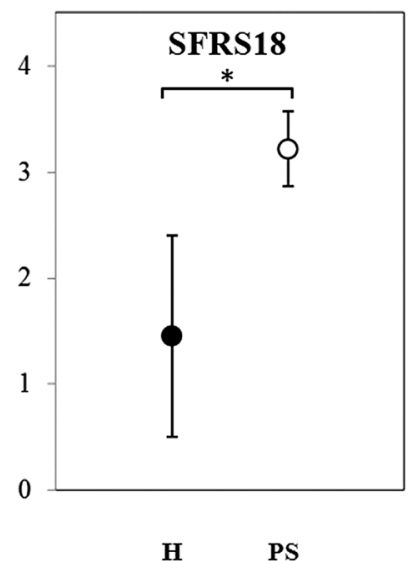



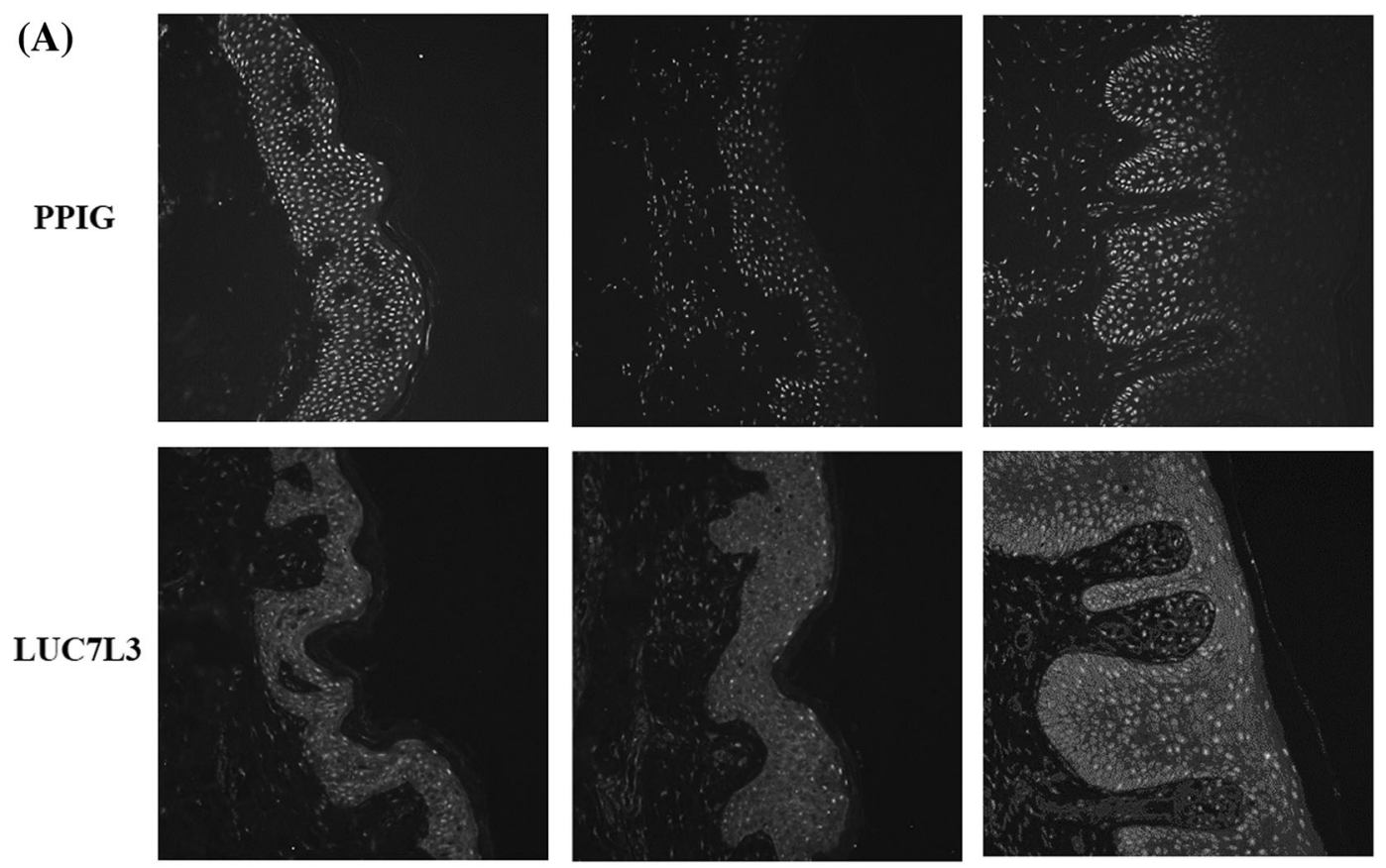

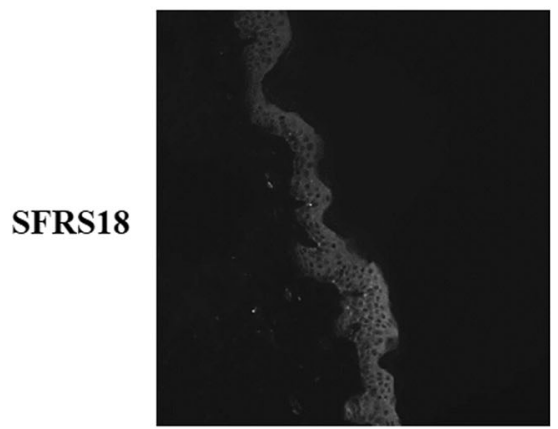

Healthy epidermis

(B)

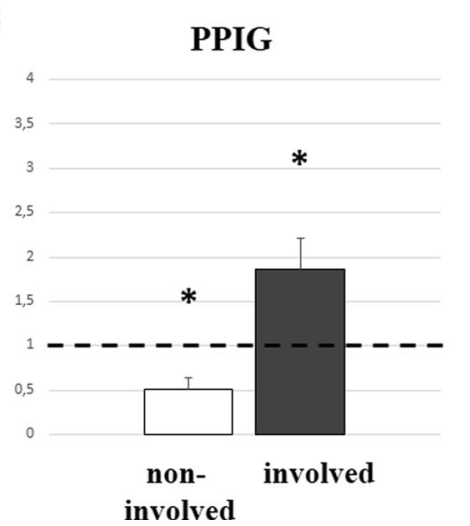

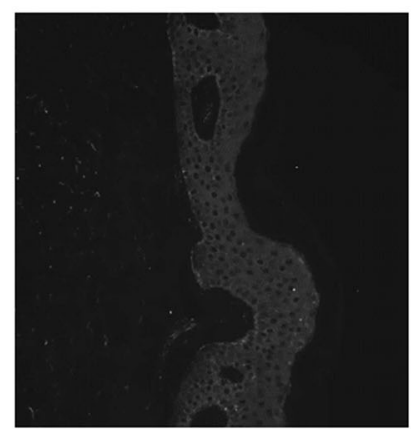

Psoriatic non-involved epidermis

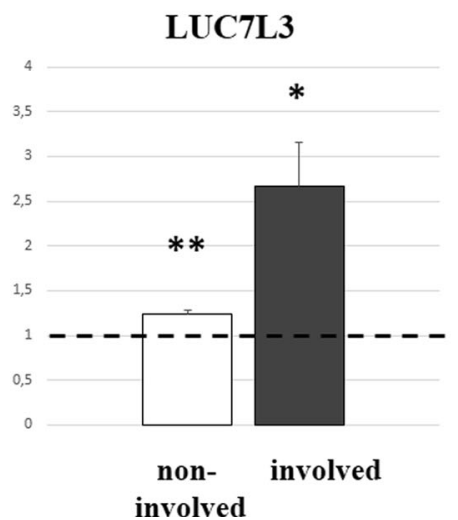

involved

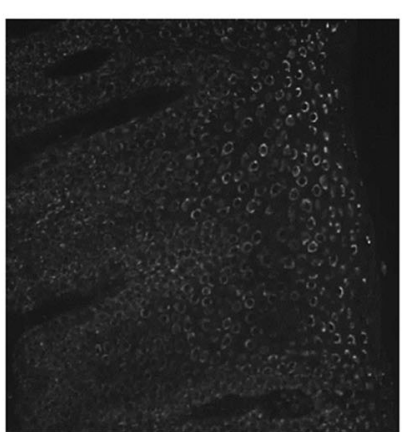

\section{Psoriatic involved} epidermis

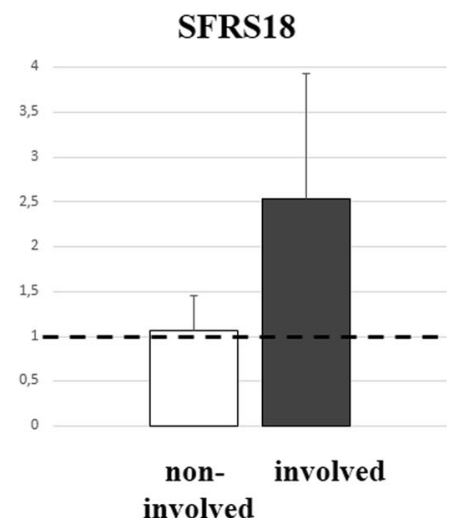

Fig. 2 Immunofluorescent staining of PPIG, LUC7L3, and SFRS18 proteins (a) was carried out on healthy $(n=3)$, psoriatic noninvolved $(n=3)$, and psoriatic involved $(n=3)$ samples. One representative staining is provided for each protein. Magnification: $\times 40$. Fluorescent intensity measurements of PPIG, LUC7L3, and
SFRS18 (b) in non-involved and involved epidermis compared to healthy samples are indicated as follows: dashed lines represent the unaltered condition, where the ratio of non-involved/healthy and involved/healthy is 1; significance levels are indicated by asterisks $(* p<0.05, * * p<0.01, * * * p<0.001)$ 

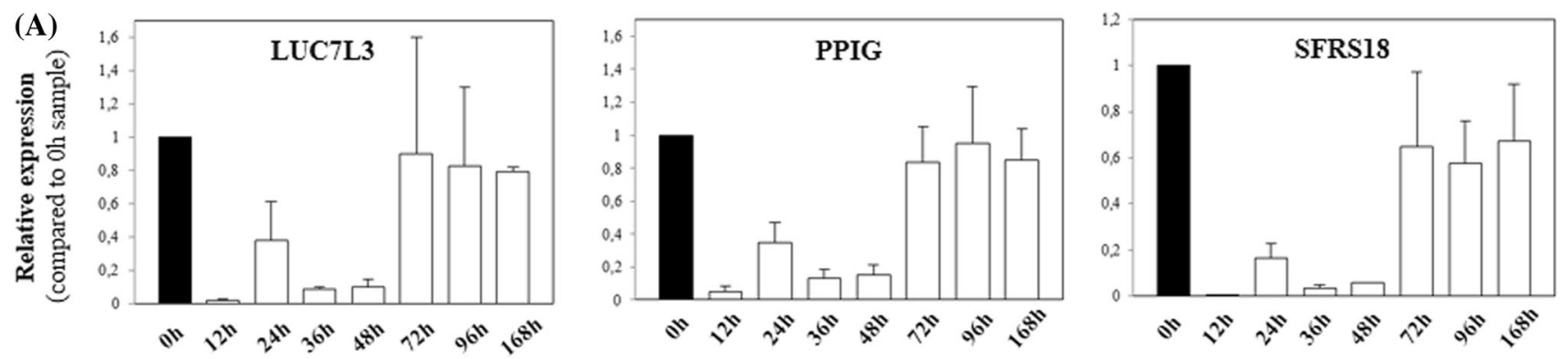

(B)
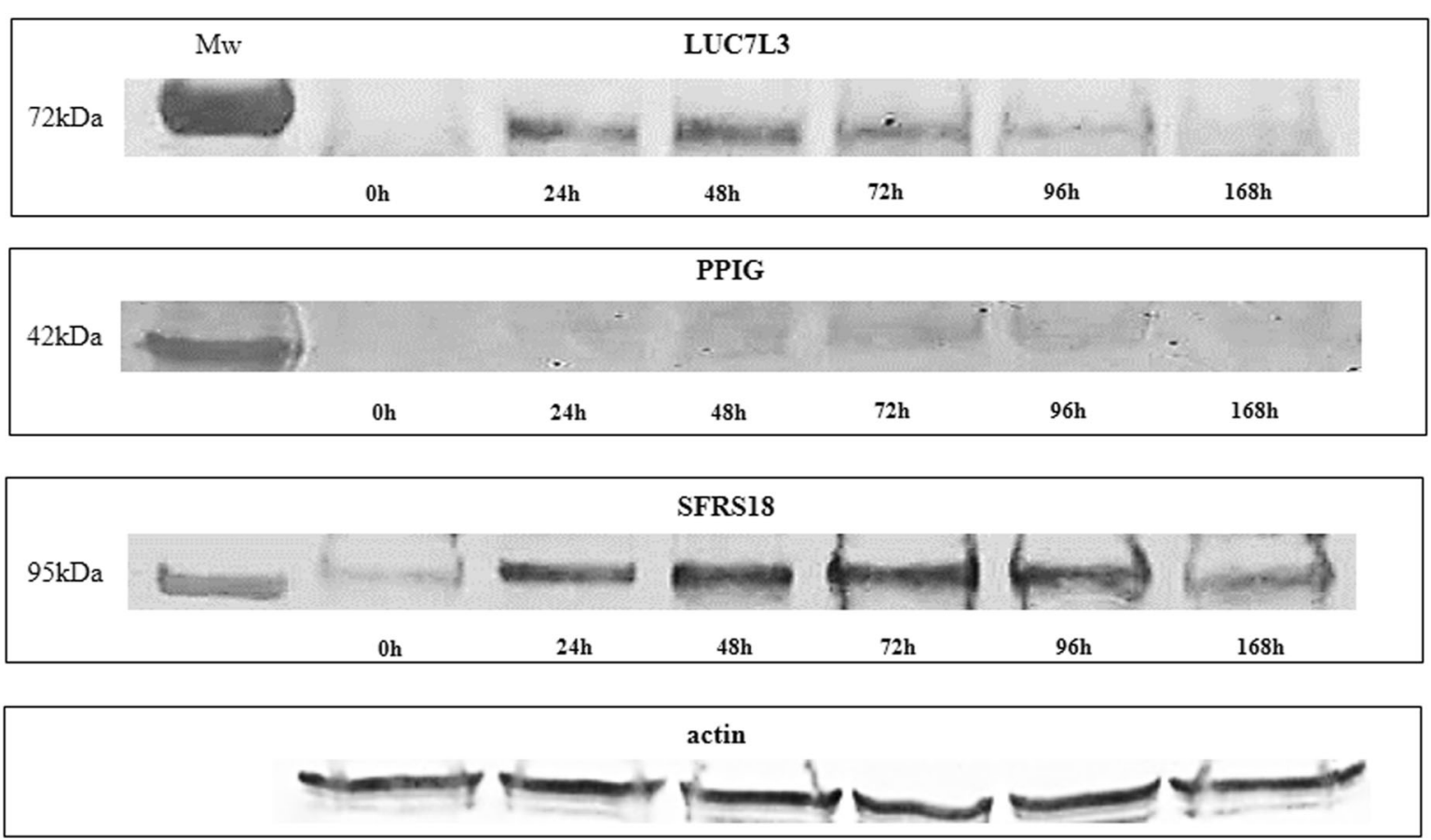

Fig. 3 HPV-KER cells were synchronized by contact inhibition and by withdrawal of supplement, and samples were taken at the indicated time points from the re-passaged $(0 \mathrm{~h})$ cells. Changes in mRNA levels (a) were followed by real-time RT-PCR and changes in protein levels (b) were followed by western blot analysis. The experiment was

\section{Silencing of PPIG, SFRS18, and $L U C 7 L 3$ affects fibronectin splicing}

To determine the physiological relevance of LUC7L3, PPIG, and SFRS18 in the fibronectin mRNA maturation processes, HPV-KER cells were transfected with siRNA constructs silencing these splicing-factor genes. The $\mathrm{EDA}+/$ total fibronectin ratios were compared in scrambled and gene-specific siRNA-transfected cells, and the mean of silencing efficacies was approximately $80 \%$ (summarized in Suppl. Fig. 2). Changes in the fibronectin splicing pattern were investigated by RT-PCR, and densitometry analysis was applied as an accurate and semiquantitative method to determine changes in the EDA+/total ratio in carried out with three independent series of cell cultures. The mRNA expression data were calculated as the average of these three parallel experiments. One representative western blot is provided for each splicing factor. $\alpha$-actin was used as a loading control

response to silencing of the three different splicing factors either alone or in combination.

In the experiments where a single splicing factor was silenced, siRNA transfection of LUC7L3 has lowered the EDA+/total ratio for fibronectin, and a slight decrease in this ratio was also seen with PPIG and SFRS18 silencing (Fig. 4a). In the scrambled RNA-transfected samples, the relative amount of the EDA+ variant was higher compared to the EDA - fibronectin isoform.

Bioinformatics analysis suggests that LUC7L3 and SFRS18 are interacting partners (Fig. 4c). Therefore, experiments in which two or three genes were silenced were also performed to examine the possible interactions among the splicing factors. The combined silencing of 


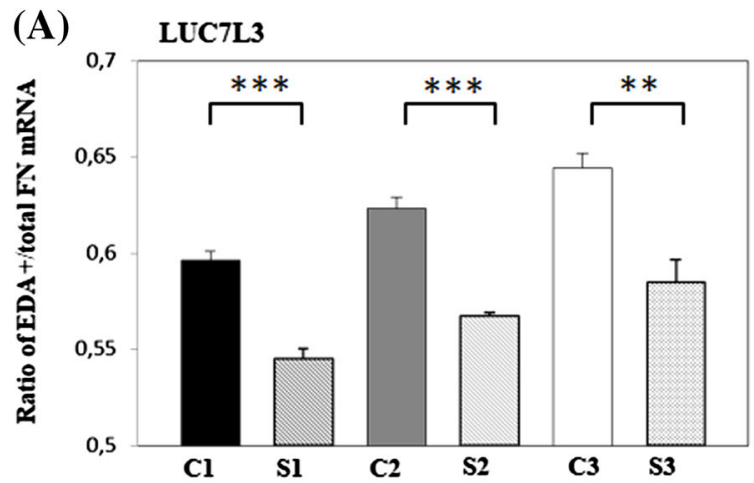

PPIG
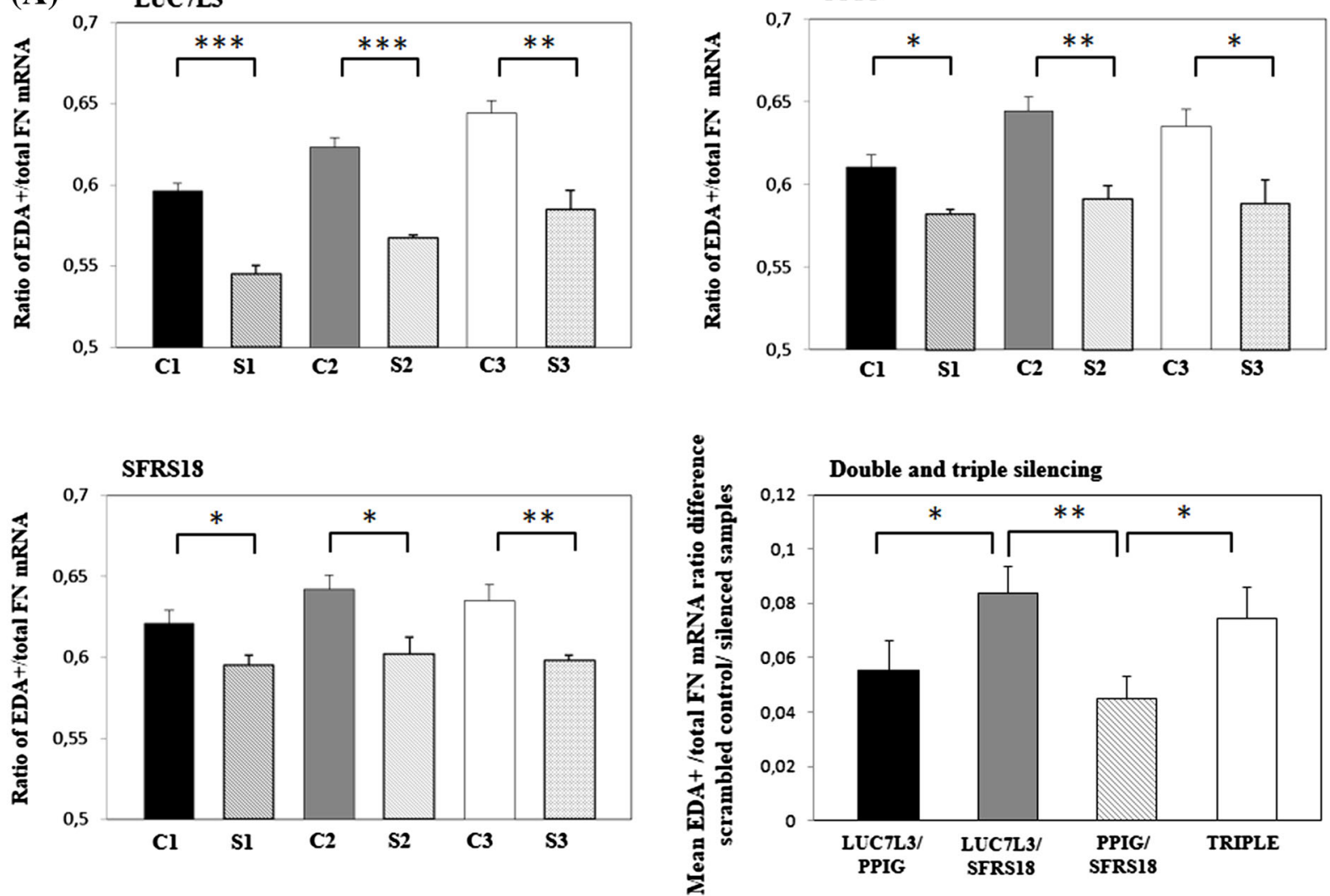

(B)

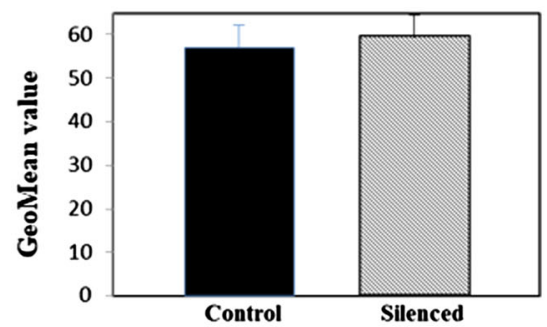

EDA+ FN

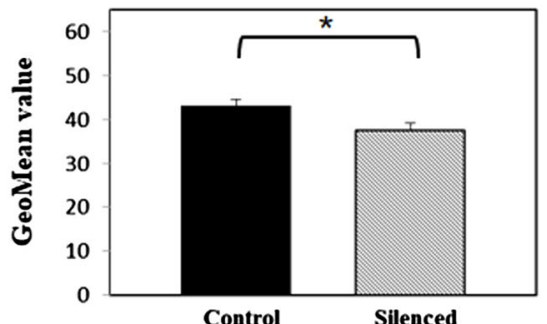

Control

Silenced

EDA+/total FN

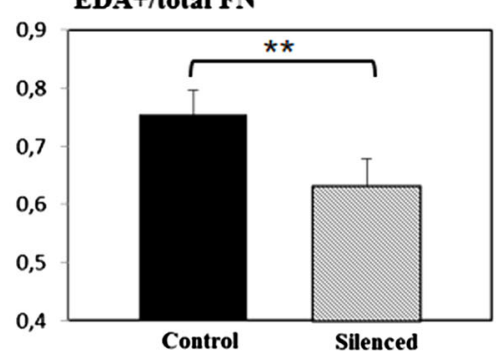

(C)

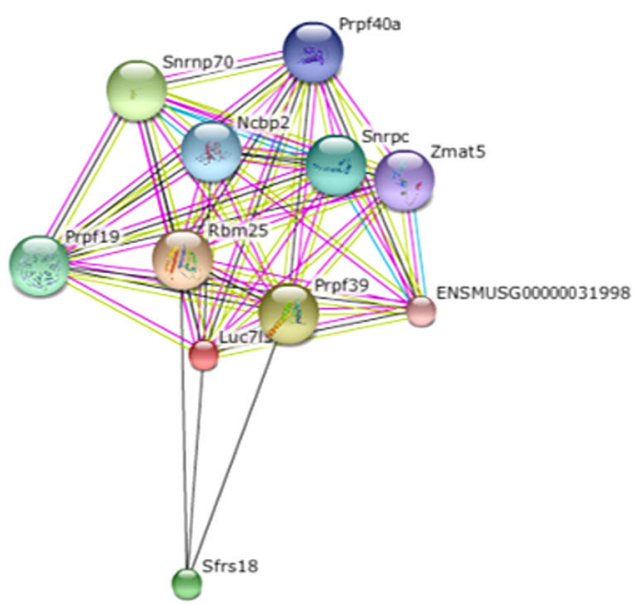

Rbm25

Prpf39

Snrnp70

Sfrs18

Prpf19

Snrpc

Ncbp2

Prpf40a

Zmat5

ENSMUSG00000031998

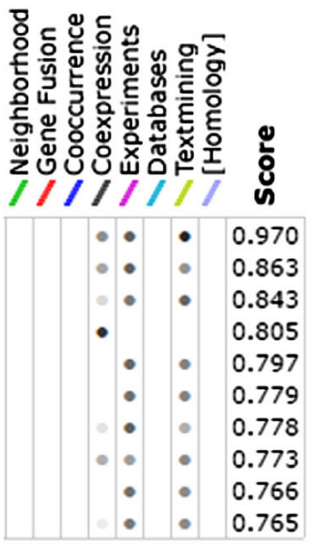


४Fig. $4 \mathrm{HPV}$-KER cells were transfected with specific silencing constructs for $L U C 7 L 3, P P I G$, and SFRS18 (a) and the ratio of EDA+ fibronectin mRNA was calculated in each repeated experiment (S1, S2, S3). The changes in this ratio were compared to that of the scrambled RNA-transfected controls (C1, C2, C3) in each experiment. Comparison of the effect of double and triple silencing of the LUC7L3, PPIG, and SFRS18 genes to the scrambled RNAtransfected controls revealed that the double silencing of $L U C 7 L 3$ and SFRS18 resulted in the most robust changes in the EDA+/total ratio of fibronectin mRNA. Effect of triple silencing did not differ significantly from that of $L U C 7 L 3$ and SFRS18 double silencing. Columns represent means of three independent experiments $(+\mathrm{SE})$, all performed in triplicate. Significance levels are indicated by asterisks $(* p<0.05, * * p<0.01, * * * p<0.001)$. Total fibronectin and EDA+ fibronectin were detected by flow cytometry in HPV-KER cells $(n=5)$ in which both LUC7L3 and SFRS18 were silenced (b). Silencing did not affect the amount of total fibronectin, whereas a significant $(p<0.05)$ decrease in the amount of the EDA+ fibronectin was detected after silencing, and this decrease resulted in significant $(p<0.01)$ changes in the EDA+/total ratio of fibronectin. Bioinformatics analysis revealed a predicted functional interaction between the LUC7L3 and SFRS18 splicing regulators (c)

LUC7L3 and SFRS18 leads to the most robust change in the EDA+/total ratio of fibronectin (Fig. 4a). Notably, silencing of all three splicing factors had no additional effect on the ratio compared to the double silencing of LUC7L3 and SFRS18.

The effect of silencing was also examined at the protein level. Total and EDA+ fibronectin amount was measured with flow cytometry using the most effective combination of double LUC7L3 and SFRS18 silencing. Similar to the mRNA experiments, the immortalized HPV-keratinocytes expressed high levels of EDA+ fibronectin protein. This double silencing significantly diminished the quantity of $\mathrm{EDA}+$ fibronectin, without decreasing the total amount of fibronectin (Fig. 4b). Taken together, these results demonstrate that silencing of the splicing factors decreased the EDA+/total fibronectin ratio both at mRNA and protein level.

\section{Discussion}

The importance of fibronectin as an excellent model for studying splicing events as well as the importance of various fibronectin isoforms-including variants containing the EDA and EDB domains-is increasingly being acknowledged. It was previously shown that EDA+ fibronectin is overexpressed in samples of psoriatic non-involved epidermis [5]. Some years later, our research group reported that both normal cultured keratinocytes and $\mathrm{HaCaT}$ cells produce the oncofetal EDA+ fibronectin isoform. Moreover, it has also been proven that keratinocytes from psoriatic non-involved epidermis are sources of EDA+ fibronectin, while the expression of this isoform is minimal in healthy keratinocytes. These data suggest that EDA+ fibronectin might initiate a signal transduction process that sensitizes keratinocytes to the mitogenic signals of immune cells [4].

In our recently performed cDNA microarray experiment, we aimed to reveal that novel molecular factors make it prone to form psoriatic lesions. From this work, we identified three splicing factors that participate in the molecular processes of mRNA maturation. In addition to regulation of splicing, PPIG is responsible for the normal folding of proteins, and LUC7L3 has been implicated in cisplatin resistance in tumor cells [27-29]. PPIG and SFRS18 are physical interactors of the splicing factor and the desmosome-associated protein, pinin [27, 30].

Our study opens the questions whether LUC7L3, PPIG, and SFRS18 are involved in the mRNA maturation process of fibronectin and whether the altered expression of these splicing factors can be linked to the documented presence of EDA+ fibronectin in psoriasis. Although EDA+ fibronectin is known to be involved in several biological processes, the regulation of its synthesis is not completely understood. TGF- $\beta$ is known to promote inclusion of EDA domain [31-33]. Other research groups reported that the SR-rich protein SF2/ASF exerts a similar effect, whereas heterogeneous ribonucleoprotein particles inhibit its inclusion $[8,34,35]$. According to the latest findings, keratinocyte growth factor (KGF) slightly increases the formation of the EDA+ variant, and STAT1 induces exclusion in fibroblasts [36].

Based on our results, LUC7L3 might have a direct regulatory role on EDA+ domain inclusion: similarly to the EDA+ fibronectin, its protein level is also increased in non-involved epidermis. SFRS18 showed only a minimal tendency towards elevation. PPIG amounts are decreased in non-involved epidermis compared to healthy controls, and this expression pattern resembles that which we previously observed for STAT1, another component of the EDA+ fibronectin-related molecular pathway. Moreover, similar to STAT1, PPIG proved to be part of the functional molecular network involving KGF and fibronectin signaling [36].

Another relevant outcome of our current study is that all three splicing factors exhibit the highest level of staining in psoriatic involved epidermis, with approximately 2- to 3 -fold elevation in involved epidermis compared to noninvolved samples. These data indicate that the examined splicing factors could influence the mRNA maturation of several other molecular components involved in the inflammatory and proliferative processes of psoriatic lesions.

In addition to tissue staining, results from in vitro characterization of splicing regulator expression also support the congruent pattern detected in psoriatic involved 
epidermis. In synchronized, immortalized keratinocytes, remarkable similarities in the expression patterns were observed both at the RNA and protein levels. Genetic linkage does not account for these similarities, as the LUC7L3, SFRS18, and PPIG genes are located on different chromosomes. Instead, the corresponding mRNAs might possess common transcription-factor binding sites and upstream regulators. Based on western blot analysis and immunofluorescent staining results, we conclude that expression of the three splicing factors is marked in both immortalized keratinocytes and involved epidermis. This finding supports that elevated amounts of LUC7L3, PPIG, and SFRS18 might be responsible for the shortened and abnormal maturation of keratinocytes that is a characteristic of psoriatic epidermis and is also typical of the immortalized HPV-KER cells.

The influence of the LUC7L3 splicing regulator on the formation of EDA+ fibronectin was also confirmed by in vitro experiments. We demonstrated that immortalized HPV-KER cells express the oncofetal EDA+ isoform of fibronectin in higher amounts than the EDA- isoform. LUC7L3 silencing resulted in the decrease of the $\mathrm{EDA}+/$ total ratio for fibronectin, indicating that the relative amount of EDA - fibronectin is elevated in response to LUC7L3 silencing. Similar results were obtained with silencing of PPIG and SFRS18, although the changes in the $\mathrm{EDA}+/$ total fibronectin ratios were more moderate than with $L U C 7 L 3$ silencing. These data suggest that the three splicing factors promote exon inclusion of the EDA domain. This finding is in accordance with previous work showing that another type of SR protein, SF2/ASF, supports the inclusion of the EDA element $[34,35]$.

Double silencing of SFRS18 and LUC7L3 was the most effective in decreasing the EDA+/total ratio of fibronectin, as expected from the results of bioinformatics analysis. Moreover, LUC7L3 and SFRS18 exhibited a similar pattern of expression during immunofluorescent staining. To analyze whether the changes in the EDA+/total fibronectin ratio detected by PCR are similar at the protein level, flow cytometric measurements were performed in cells in which both SFRS 18 and LUC7L3 were silenced. The EDA+/total ratio for fibronectin protein decreased in these experiments. The quantity of total fibronectin was constant before and after silencing, and the ratio changes were thus due to increased production of the EDA - fibronectin isoform via an altered splicing mechanism. In addition to SF2/ASF, other SR-rich proteins have also been shown to promote inclusion of the EDA domain. This finding is of special interest, considering that SR proteins usually interact with enhancer splicing elements, inducing exon inclusion [37-39].

Several previous studies indicate that alternative splicing changes the binding characteristics of fibronectin radically $[6,8,13,40]$. The $\alpha 5 \beta 1$ integrin receptor is one of the most important interacting partners for fibronectin, and the inclusion of the EDA domain has been shown to increase affinity towards this receptor [6, 40, 41]. However, a contradicting report demonstrated that the EDA domain does not bind to $\alpha 5 \beta 1$ integrin [8]. It is also well documented that EDA+ fibronectin binds to the TLR4 receptor, which is part of the innate immune system $[6,33,42]$. It would be a very interesting new approach to study the changes of TLR4-mediated signal transduction in response to the altered expression of splicing factors.

Taken together, LUC7L3 might- through inclusion of the EDA domain and, thus, elevation of EDA+ fibronectin abundance in the epidermis — contribute to an autocrine loop by which psoriatic keratinocytes maintain hyper-responsiveness towards proliferative signals. Although the amounts of SFRS18 did not show elevation in the non-involved epidermis, in vitro studies support the suggestion that the presence of this protein could facilitate the effect of LUC7L3. All three splicing regulators are notably elevated in psoriatic involved epidermis, indicating that the mRNA maturation of fibronectin is influenced by these proteins, and that these regulators could also have multiple roles in disease development.

In future work, it would be interesting to clarify the upstream regulatory elements maintaining the highly synchronous expression of these three splicing factors and whether these elements are altered in psoriasis. Moreover, identification of additional mRNAs with altered expression and/or splicing patterns under the regulation of the LUC7L3, PPIG, and SFRS18 splicing factors might lead to the identification of novel molecular patterns in psoriasis pathogenesis.

Acknowledgements The study was supported by OTKA K105985, OTKA K111885 and TÁMOP-4.2.2.A-11/1/KONV-2012-0035, GINOP-2.3.2-15-2016-00015 research Grants.

\section{References}

1. Nestle FO, Kaplan DH, Barker J (2009) Psoriasis. N Engl J Med 361:496-509

2. Roberson D, Bowcock AM (2010) Psoriasis genetics: breaking the barrier. Trends Genet 26:415-423

3. Lowes MA, Suarez-Farinas M, Krueger JG (2014) Immunology of psoriasis. Annu Rev Immunol 32:227-255

4. Szell M, Bata-Csorgo Z, Koreck A, Pivarcsi A, Polyanka H, Szeg C, Gaal M, Dobozy A, Kemeny L (2004) Proliferating keratinocytes are putative sources of the psoriasis susceptibility-related EDA+ (extra domain A of fibronectin) oncofetal fibronectin. J Invest Dermatol 123:537-546

5. Ting KM, Rothaupt D, McCormick TS, Hammerberg C, Chen G, Gilliam AC, Stevens S, Culp L, Cooper KD (2000) Overexpression of the oncofetal Fn variant containing the EDA splice-in segment in the dermal-epidermal junction of psoriatic uninvolved skin. J Invest Dermatol 114:706-711

6. McFadden JP, Basketter DA, Dearman RJ, Kimber IR (2011) Extra domain A-positive fibronectin-positive feedback loops and 
their association with cutaneous inflammatory disease. Clin Dermatol 29:257-265

7. Kornblihtt AR, Pesce CG, Alonso CR, Cramer P, Srebrow A, Werbajh S, Muro AF (1996) The fibronectin gene as a model for splicing and transcription studies. FASEB J 10:248-257

8. White ES, Baralle FE, Muro AF (2008) New insights into form and function of fibronectin splice variants. J Pathol 216:1-14

9. Ffrench-Constant C, Van de Water L, Dvorak HF, Hynes RO (1989) Reappearance of an embryonic pattern of fibronectin splicing during wound healing in the adult rat. J Cell Biol 109:903-914

10. Lohi J, Tani T, Laitinen L, Kangas L, Lehto VP, Virtanen I (1995) Tenascin and fibronectin isoforms in human renal cell carcinomas, renal cell carcinoma cell lines and xenografts in nude mice. Int J Cancer 63:442-449

11. Astrof S, Crowley D, George EL, Fukuda T, Sekiguchi K, Hanahan D, Hynes RO (2004) Direct test of potential roles of EIIIA and EIIIB alternatively spliced segments of fibronectin in physiological and tumor angiogenesis. Mol Cell Biol 24:8662-8670

12. White ES, Muro AF (2011) Fibronectin splice variants: understanding their multiple roles in health and disease using engineered mouse models. IUBMB Life 63:538-546

13. Xu J, Mosher D (2011) The extracellular matrix: an overview. Biology of extracellular matrix. Springer, Heidelberg, pp 41-76

14. Baechler EC, Batliwalla FM, Reed AM, Peterson EJ, Gaffney PM, Moser KL, Gregersen PK, Behrens TW (2006) Gene expression profiling in human autoimmunity. Immunol Rev 210:120-137

15. Romanowska M, Yacoub N, Seidel H, Donandt S, Gerken H, Phillip S, Haritonova N, Artuc M, Schweiger S, Sterry W, Foerster J (2008) PPARdelta enhances keratinocyte proliferation in psoriasis and induces heparin-binding EGF-like growth factor. J Invest Dermatol 128:110-124

16. Gudjonsson JE, Ding J, Li X, Tejasvi T, Qin ZS, Ghosh D, Aphale A, Gumucio DL, Voorhees JJ, Abecasis GR, Elder JT (2009) Global gene expression analysis reveals evidence for decreased lipid biosynthesis and increased innate immunity in uninvolved psoriatic skin. J Invest Dermatol 129:2795-2804

17. Blumenberg M (2012) SKINOMICS: transcriptional profiling in dermatology and skin biology. Curr Genomics 13:363-368

18. Manczinger M, Kemeny L (2013) Novel factors in the pathogenesis of psoriasis and potential drug candidates are found with systems biology approach. PLoS ONE 8:e80751

19. Villasenor-Park J, Ortega-Loayza AG (2013) Microarray technique, analysis, and applications in dermatology. J Invest Dermatol 133:e7

20. Keermann M, Koks S, Reimann E, Prans E, Abram K, Kingo K (2015) Transcriptional landscape of psoriasis identifies the involvement of IL36 and IL36RN. BMC Genomics 16:322

21. Li B, Tsoi LC, Swindell WR, Gudjonsson JE, Tejasvi T, Johnston A, Ding J, Stuart PE, Xing X, Kochkodan JJ, Voorhees JJ, Kang HM, Nair RP, Abecasis GR, Elder JT (2014) Transcriptome analysis of psoriasis in a large case-control sample: RNA-seq provides insights into disease mechanisms. J Invest Dermatol 134:1828-1838

22. Koks S, Keermann M, Reimann E, Prans E, Abram K, Silm H, Koks G, Kingo K (2016) Psoriasis-specific RNA isoforms identified by RNA-seq analysis of 173,446 transcripts. Front Med (Lausanne) 3:46

23. Szabo K, Bata-Csorgo Z, Dallos A, Bebes A, Francziszti L, Dobozy A, Kemeny L, Szell M (2014) Regulatory networks contributing to psoriasis susceptibility. Acta Derm Venereol 94:380-385

24. Bata-Csorgo Z, Hammerberg C, Voorhees JJ, Cooper KD (1995) Kinetics and regulation of human keratinocyte stem cell growth in short-term primary ex vivo culture. Cooperative growth factors from psoriatic lesional $\mathrm{T}$ lymphocytes stimulate proliferation among psoriatic uninvolved, but not normal, stem keratinocytes. J Clin Invest 95:317-327

25. Polyanka H, Szabo K, Tax G, Tubak V, Kusz E, Ujfaludi Z, Boros I, Bata-Csorgo Z, Kemeny L, Szell M (2011) Primary characterization of a novel HPV- E6 oncogene immortalized keratinocyte cell line. J Invest Dermatol 131(Suppl. 2):S70

26. Pivarcsi A, Szell M, Kemeny L, Dobozy A, Bata-Csorgo Z (2001) Serum factors regulate the expression of the proliferationrelated genes alpha5 integrin and keratin 1, but not keratin 10, in HaCaT keratinocytes. Arch Dermatol Res 293:206-213

27. Lin CL, Leu S, Lu MC, Ouyang P (2004) Over-expression of SRcyclophilin, an interaction partner of nuclear pinin, releases SR family splicing factors from nuclear speckles. Biochem Biophys Res Commun 321:638-647

28. Nishii Y, Morishima M, Kakehi Y, Umehara K, Kioka N, Terano Y, Amachi T, Ueda K (2000) CROP/Luc7A, a novel serine/ arginine-rich nuclear protein, isolated from cisplatin-resistant cell line. FEBS Lett 465:153-156

29. Puig O, Bragado-Nilsson E, Koski T, Seraphin B (2007) The U1 snRNP-associated factor Luc7p affects $5^{\prime}$ splice site selection in yeast and human. Nucleic Acids Res 35:5874-5885

30. Zimowska G, Shi J, Munguba G, Jackson MR, Alpatov R, Simmons MN, Shi Y, Sugrue SP (2003) Pinin/DRS/memA interacts with SRp75, SRm300 and SRrp130 in corneal epithelial cells. Invest Ophthalmol Vis Sci 44:4715-4723

31. Balza E, Borsi L, Allemanni G, Zardi L (1988) Transforming growth factor beta regulates the levels of different fibronectin isoforms in normal human cultured fibroblasts. FEBS Lett 228:42-44

32. Borsi L, Castellani P, Risso AM, Leprini A, Zardi L (1990) Transforming growth factor-beta regulates the splicing pattern of fibronectin messenger RNA precursor. FEBS Lett 261:175-178

33. Kelsh RM, McKeown-Longo PJ, Clark RA (2015) EDA fibronectin in keloids create a vicious cycle of fibrotic tumor formation. J Invest Dermatol 135:1714-1718

34. Caputi M, Casari G, Guenzi S, Tagliabue R, Sidoli A, Melo CA, Baralle FE (1994) A novel bipartite splicing enhancer modulates the differential processing of the human fibronectin EDA exon. Nucleid Acids Res 22:1018-1022

35. Buratti E, Muro AF, Giombi Gherbassi D, Iaconcig A, Baralle FE (2004) RNA folding affects the recruitment of SR proteins by mouse and human polypurinic enhancer elements in the fibronectin EDA exon. Mol Cell Biol 24:1387-1400

36. Guban B, Vas K, Balog Z, Manczinger M, Bebes A, Groma G, Szell M, Kemeny L, Bata-Csorgo Z (2016) Abnormal regulation of fibronectin production by fibroblasts in psoriasis. Br J Dermatol 174:533-541

37. Faustino NA, Cooper TA (2003) Pre-mRNA splicing and human disease. Genes Dev 17:419-437

38. Pagani F, Baralle FE (2010) Analysis of human splicing defects using hybrid minigenes. Molecular diagnostics. Elsevier, New York, pp 155-169

39. Ward AJ, Cooper TA (2011) The pathobiology of splicing. J Pathol 220:152-163

40. Pankov R, Yamada KM (2002) Fibronectin at glance. J Cell Sci 115:3861-3863

41. Pellegrini G, De Luca M, Orecchia G, Balzac F, Cremona O, Savoia P, Cancedda R, Marchisio PC (1992) Expression, topography, and function of integrin receptors are severely altered in keratinocytes from involved and uninvolved psoriatic skin. J Clin Invest 89:1783-1795

42. Okamura KY, Watari M, Jerud ES, Young DW, Ishizaka ST, Rose J, Chow JC, Strauss JF (2002) The extra domain A of fibronectin activates Toll-like receptor 4. J Biol Chem 276:10229-10233 